\title{
BMJ Open Relationship between osteonecrosis and antiphospholipid antibodies in patients with systemic lupus erythematosus: a systematic review protocol
}

Qijiao Wei (1) , ${ }^{1,2}$ Meng Zhou, ${ }^{1,2}$ Jianwen Liu, ${ }^{1,2}$ Shengli Zhang, ${ }^{1,2}$ Fei Gao, ${ }^{1,2}$ He Lin, ${ }^{1,2}$ Zhihan Chen ${ }^{1,2}$

To cite: Wei Q, Zhou M, Liu J, et al. Relationship between osteonecrosis and antiphospholipid antibodies in patients with systemic lupus erythematosus: a systematic review protocol. BMJ Open 2021;11:e046163. doi:10.1136/ bmjopen-2020-046163

- Prepublication history for this paper is available online. To view these files, please visit the journal online (http://dx.doi. org/10.1136/bmjopen-2020046163).

Received 25 0ctober 2020 Accepted 07 July 2021

Check for updates

(c) Author(s) (or their employer(s)) 2021. Re-use permitted under CC BY-NC. No commercial re-use. See rights and permissions. Published by BMJ.

${ }^{1}$ Rheumatology, Fujian Medical University Provincial Clinical Medical College, Fuzhou, Fujian, China

${ }^{2}$ Rheumatology, Fujian Provincial Hospital, Fuzhou, Fujian, China

Correspondence to

Dr Zhihan Chen;

han213@163.com

\section{ABSTRACT}

Introduction 0steonecrosis (ON) is characterised by the destruction of the normal blood supply to the bone tissue. $\mathrm{ON}$ is the main cause of disability in patients with systemic lupus erythematosus (SLE). Studies have reported the existence of many risk factors for SLE complicated by ON, including the use of high-dose glucocorticoids and high disease activity. The correlation between antiphospholipid antibodies (aPLs) and ON in SLE has been controversial. We aim to conduct a systematic review of the literature related to SLE, aseptic ON and aPLs, to provide a reference for the clinical screening of high-risk patients and for early prevention.

Methods and analysis The following six databases will be searched: MEDLINE/PubMed, Embase, Web of Science, Chinese Biomedical Literature Database, Wan-Fang Database and China National Knowledge Infrastructure. The database searches will not be restricted by date. Case-control studies, cohort studies or observational studies that compare aPLs between SLE patients with and without ON will be considered eligible. Articles published in English and Chinese will be included. Two researchers will independently perform the processes of study selection, data extraction and study quality assessment. The Newcastle-0ttawa Quality Assessment Scale will be used to assess the quality of the retrieved studies. A metaanalysis will be performed after screening the studies. Data will be analysed using ORs for dichotomous data. Ethics and dissemination Ethical approval is not required because this systematic review will use published data. The systematic review will be electronically disseminated through a peer-reviewed publication or conference presentations.

PROSPERO registration number CRD42020209637.

\section{INTRODUCTION}

Osteonecrosis $(\mathrm{ON})$ is characterised by the destruction of the normal blood supply to the bone tissue for various reasons, leading to the collapse of the bone structure and causing joint pain and loss of function. The commonly affected parts are the femoral head and knee joints. ${ }^{1} \mathrm{ON}$ is the main cause of disability in patients with systemic lupus erythematosus
Strengths and limitations of this study

- This study will systematically report the relationship among systemic lupus erythematosus, aseptic osteonecrosis and different antiphospholipid antibodies, to provide a reference for the clinical screening of high-risk patients and for early prevention.

- The quality of the studies will be carefully assessed using the Newcastle-0ttawa Quality Assessment Scale.

- Different study designs may cause considerable heterogeneity, which could limit the generation of convincing conclusions.

(SLE), seriously affecting their quality of life. ${ }^{2}$ The use of high-dose glucocorticoids is a definite, but not the only, risk factor for ON. Studies have reported the existence of other risk factors for SLE complicated by ON. ${ }^{3}{ }^{4}$ Antiphospholipid antibodies (aPLs) include lupus anticoagulant (LA), anticardiolipin (ACL) and anti-beta 2 glycoprotein

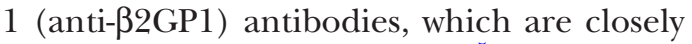
related to thrombus formation. ${ }^{5}$ The correlation between aPLs and ON in SLE has been controversial. On the basis of the previous findings and the fact that no meta-analysis has been conducted, we aim to conduct a meta-analysis of the literature related to SLE, aseptic ON and aPLs to provide a reference for the clinical screening of high-risk patients and for early prevention.

\section{METHODS AND ANALYSIS}

The protocol has been registered with PROSPERO (International Prospective Register of Systematic Reviews). We will conform to the Preferred Reporting Items for Systematic Reviews and Meta-analyses guidelines for the development of the systematic review. 


\section{Research question}

This systematic review aims to answer the following question: What is the relationship between aPLs and ON in SLE? We only explore the relationship between the positive rate of aPLs and ON, not involving the titre of aPLs.

\section{Patient and public involvement}

No patients will be involved.

\section{Criteria for inclusion of studies in this review \\ Types of studies}

Observational, case-control, cohort and cross-sectional studies will be included. Case reports and cases series (with fewer than five patients) will be excluded.

\section{Types of participants}

Patients with SLE will be included without age limitations. Studies related to aPLs in SLE combined with aseptic ON published in English or Chinese will be included. Cases should have clear diagnostic criteria. The diagnosis of SLE should have been made according to the 1982 revised American Rheumatism Association criteria, 1997 American College of Rheumatology classification criteria, Systemic Lupus International Collaborating Clinics classification criteria or 2019 SLE European League Against Rheumatism/American College of Rheumatology classification criteria. ON should have been radiologically confirmed using plain radiography, bone scans, MRI or tomography. Patients with other rheumatologic diseases, including Takayasu arteritis, rheumatoid arthritis and ankylosing spondylitis, will be excluded. Patients with other types of $\mathrm{ON}$, such as traumatic $\mathrm{ON}$ and infectious $\mathrm{ON}$, will be excluded.

Types of outcome assessments

Studies should provide clear regulations on the sample size. Each article must provide the OR and 95\% CI for positivity of any aPL.

\section{Search methods for identification of studies}

The search strategy will be applied according to the Cochrane Handbook guidelines. ${ }^{6}$ The following six databases will be searched from their inception through 26 December 2020: MEDLINE/PubMed, Embase, Web of Science, Chinese Biomedical Literature Database, WanFang Database and China National Knowledge Infrastructure. The keywords will include the following: "Systemic lupus erythematosus", "Lupus Erythematosus Disseminatus", "Libman-Sacks Disease", "Libman Sacks Disease", "Osteonecrosis", "Bone Necrosis", "Avascular Necrosis of Bone", "Bone Avascular Necrosis", "Kienbock Disease", "Kienboeck's Disease", "kienboecks disease", "Aseptic Necrosis of Bone" and "Bone Aseptic Necrosis." The strategy for searching the PubMed database is shown in table 1. This search strategy will also be applied to the other electronic databases.

\section{Screening and data collection}

The titles and abstracts will be screened by two authors. Any disagreement during the selection of studies will be

\begin{tabular}{ll}
\hline Table 1 & Search strategy used in the PubMed database \\
\hline No. & Search items \\
\hline 1 & Systemic lupus erythematosus \\
\hline 2 & Lupus Erythematosus Disseminatus \\
\hline 4 & Libman-Sacks Disease \\
\hline 5 & Libman Sacks Disease \\
\hline 6 & or 1-4 \\
\hline 7 & Osteonecrosis \\
\hline 8 & Bone Necrosis \\
\hline 9 & Avascular Necrosis of Bone \\
\hline 10 & Bone Avascular Necrosis \\
11 & Kienbock Disease \\
\hline 12 & Kienboeck's Disease \\
\hline 13 & kienboecks disease \\
\hline 14 & Aseptic Necrosis of Bone \\
\hline 15 & Bone Aseptic Necrosis \\
\hline 16 & or 6-14 \\
\hline
\end{tabular}

This search strategy will be modified as required for other electronic databases.

discussed and decided by a third author. The details of the selection process are shown in the flowchart in figure 1. The results will be screened by checking the titles and abstracts of the articles. Studies pertaining to ON, SLE and aPLs will be included. Studies that compare the positive rate of aPLs between $\mathrm{ON}$ and non-ON groups will be included.

\section{Quality assessment and data extraction}

The quality of the included studies will be assessed using the Newcastle-Ottawa Quality Assessment Scale. Scores between 7 and the maximum of 9 will be defined as high quality; scores between 4 and 6 will be defined as intermediate quality and scores between 1 and 3 will be defined as low quality. Two authors will independently extract the following data: general information (name, country, year of publication, title of the study, authors' publication details, number of patients, and mean age and range), study characteristics and follow-up periods (from the measurement of the antibodies to the onset of $\mathrm{ON}$, from the measurement of the antibodies to the end of follow-up, from the onset of $\mathrm{ON}$ to the end of follow-up). All searched studies will be imported to EndNote software, which can assist the reviewers in managing data and screening for duplicate publications. The data will subsequently be entered into Stata V.11.0 software for analysis. Any disagreement will be discussed and finally decided by a third author.

\section{Analysis methods}

Raw numerical data will be extracted from the studies. A meta-analysis will be performed, if possible, using Stata V.11.0 software. Before combining the statistics, tests for heterogeneity will be conducted. If $p>0.1$, the 

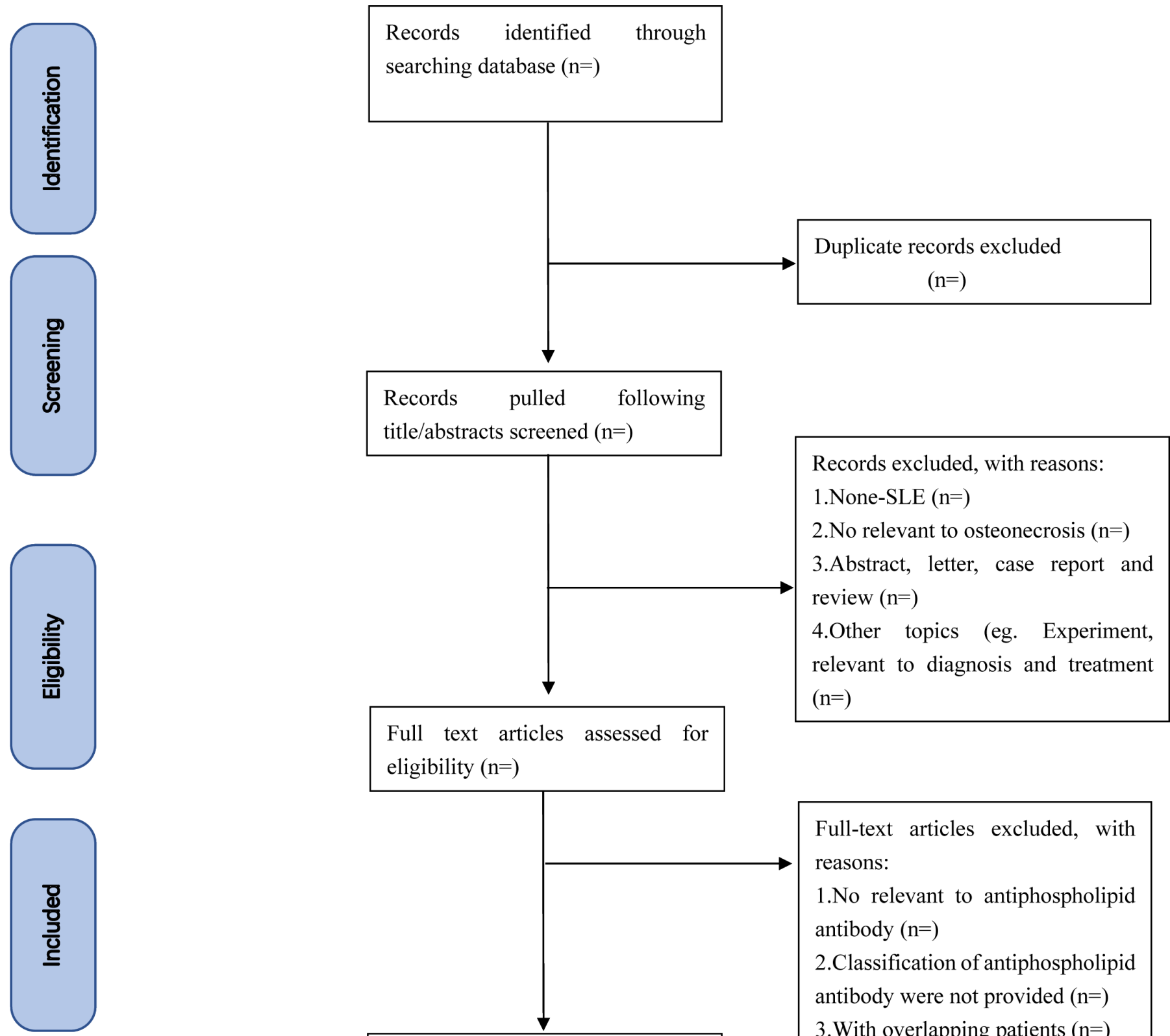

Full text articles assessed for eligibility $(n=)$

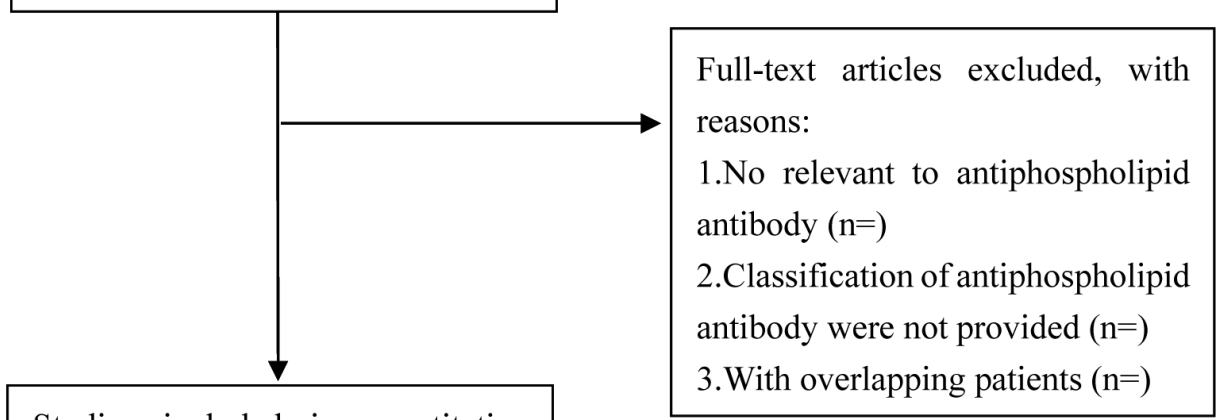

Studies included in quantitative synthesis $(\mathrm{n}=)$

Figure 1 Study flow diagram. SLE, systemic lupus erythematosus.

fixed-effects model will be used to combine the data. If $\mathrm{p}<0.1$, the DerSimonian and Laird random-effect model will be used. The reasons for heterogeneity will be analysed, and subgroup analysis will be performed. The subgroup analysis will be performed according to the study design, country of origin of the participants, age of the participants and sample size, if necessary. The results will be expressed as ORs with 95\% CIs. Two of the authors will independently assess the studies and perform adjustments for risk of bias. Harbord's modified test and funnel plots will be used to assess reporting biases.

Because our search will include studies across different settings, other factors such as glucocorticoid use or hyperlipidaemia may confound the independent effect of aPLs on ON in SLE. To exclude the independent effect, we will distinguish between descriptive studies and those that adjusted for independent effects.

We hope to complete the entire study by the end of May 2021.

\section{DISCUSSION}

aPLs include LA, ACL and $\beta 2 \mathrm{GP} 1$. The correlation between ON and aPLs in patients with SLE was first proposed by Asherson. Since then, many studies have investigated this association. However, the results have been inconsistent and inconclusive. ${ }^{78}$ Therefore, we aim 
to review the literature and to perform a meta-analysis to derive more precise results.

Previous studies have shown that ACL, anti- $\beta 2 \mathrm{GP} 1$ and LA antibodies are highly related to thrombosis. ${ }^{9} 10$ Immune complexes and aPLs in patients with SLE are important factors that cause blood coagulation. ${ }^{11}$ At present, it is believed that $\mathrm{ON}$ is a comprehensive result of metabolic and local factors affecting blood supply. ${ }^{12} 13$ Meanwhile, thrombosis is a risk factor for ON. ${ }^{14}{ }^{15}$

The primary objective of this systematic review is to evaluate the correlation between ON and aPLs in patients with SLE. We will conduct qualitative and quantitative analyses of the overall data of each study to determine the relationship between aPLs and ON in patients with SLE and, consequently, to provide a reference for the clinical screening of high-risk patients and for early prevention.

In conclusion, this will be the first systematic review to assess the association between aPLs and $\mathrm{ON}$ in patients with SLE. The findings from this review might clarify the current issues on aPLs and ON in SLE, and may help experts and clinicians reach a consensus and develop guidelines to minimise problems and optimise patient outcomes.

\section{Ethics and dissemination}

Ethical approval and patient consent are not required because the results of this systematic review will be disseminated through publication in a peer-reviewed journal and presented at a relevant conference. The data used in this systematic review will not contain individual patient data. The study commenced at 26 December 2020, and its expected completion date is the end of May 2021. This study aims at reporting the relationship among SLE, aseptic ON and different aPLs, to provide a reference for the clinical screening of high-risk patients and for early prevention.

Acknowledgements The authors acknowledge the assistance provided by Fujian Provincial Hospital Library in conducting the literature search.

Contributors WQJ prepared the first draft. CZH designed the systematic review protocol and reviewed and revised the first draft. ZM designed the search strategy and will perform the search. LJW and ZM will be included in the study screening to extract data and assess the risk of bias in the included studies. GF, ZSL and CZH will dispute disagreements between reviewers. LH will analyse and interpret the data.

Funding This work was supported by Natural Science Foundation of Fujian Province, Grant No. 2019J01184.

Competing interests None declared.
Patient and public involvement Patients and/or the public were not involved in the design, or conduct, or reporting, or dissemination plans of this research.

Patient consent for publication Not required.

Provenance and peer review Not commissioned; externally peer reviewed.

Open access This is an open access article distributed in accordance with the Creative Commons Attribution Non Commercial (CC BY-NC 4.0) license, which permits others to distribute, remix, adapt, build upon this work non-commercially, and license their derivative works on different terms, provided the original work is properly cited, appropriate credit is given, any changes made indicated, and the use is non-commercial. See: http://creativecommons.org/licenses/by-nc/4.0/.

ORCID iD

Qijiao Wei http://orcid.org/0000-0002-7791-0630

\section{REFERENCES}

1 Matthews AH, Davis DD, Fish MJ. Osteonecrosis (Avascular Necrosis). In: StatPearls (Internet). Treasure Island (FL): StatPearls Publishing, 2020.

2 Hussein S, Suitner M, Béland-Bonenfant S, et al. Monitoring of osteonecrosis in systemic lupus erythematosus: a systematic review and metaanalysis. J Rheumatol 2018;45:1462-76.

3 Zhang K, Zheng Y, Jia J, et al. Systemic lupus erythematosus patients with high disease activity are associated with accelerated incidence of osteonecrosis: a systematic review and meta-analysis. Clin Rheumatol 2018;37:5-11.

4 Nevskaya T, Gamble MP, Pope JE. A meta-analysis of avascular necrosis in systemic lupus erythematosus: prevalence and risk factors. Clin Exp Rheumatol 2017;35:700-10.

5 Mezhov V, Segan JD, Tran H, et al. Antiphospholipid syndrome: a clinical review. Med J Aust 2019;211:184-8.

6 Cumpston M, Li T, Page MJ, et al. Updated guidance for trusted systematic reviews: a new edition of the Cochrane Handbook for systematic reviews of interventions. Cochrane Database Syst Rev 2019;10:ED000142.

7 Abu-Shakra M, Buskila D, Shoenfeld Y. Osteonecrosis in patients with SLE. Clin Rev Allergy Immunol 2003;25:13-24.

8 Mukherjee S, Culliford D, Arden N, et al. What is the risk of having a total hip or knee replacement for patients with lupus? Lupus 2015;24:198-202.

9 Moulis G, Audemard-Verger A, Arnaud L, et al. Risk of thrombosis in patients with primary immune thrombocytopenia and antiphospholipid antibodies: a systematic review and meta-analysis. Autoimmun Rev 2016;15:203-9.

10 Tektonidou MG, Moutsopoulos HM. Osteoarticular manifestations of antiphospholipid syndrome. Rheum Dis Clin North Am 2006;32:523-35.

11 Adams MJ, Palatinus AA, Harvey AM, et al. Impaired control of the tissue factor pathway of blood coagulation in systemic lupus erythematosus. Lupus 2011;20:1474-83.

12 Abeles M, Abeles AM, Urman J, et al. Osteonecrosis in SLE. Rheumatol Int 2012;32:833.

13 Lespasio MJ, Sodhi N, Mont MA. Osteonecrosis of the hip: a primer. Perm J 2019;23:18-100.

14 Glueck CJ, Freiberg RA, Wang P. Role of thrombosis in osteonecrosis. Curr Hematol Rep 2003;2:417-22.

15 Masuyama J, Umino T, Murata M, et al. [A systemic lupus erythematosus patient with multiple aseptic bone necroses, thrombosis of superior mesenteric artery and anti-phospholipid antibody]. Ryumachi 1992;32:502-7. 